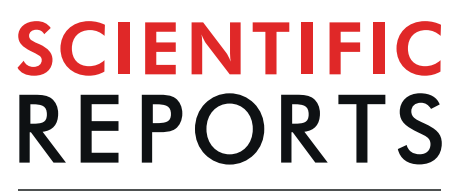

natureresearch

\title{
Type 2 diabetes-associated single nucleotide polymorphism in Sorcs1 gene results in alternative processing of the Sorcs1 protein in INS1 $\beta$-cells
}

\author{
Belinda Yau, Zachary Blood, Yousun An, Zhiduan Su \& Melkam A. Kebede $\mathbb{1}^{*}$
}

A threonine-to-Isoleucine ( $\mathrm{Thr}_{52} \mathrm{Ile}$ ) mutation within the pro-domain of the Sorcs1 gene was positionally cloned as the gene underlying a quantitative trait locus that affects fasting insulin levels in mice. In humans, genome-wide association studies and linkage studies have shown that SORCS1 is associated with diabetes and all of diabetes complications. We have recently shown that deletion of Sorcs 1 in mice made obese with the leptin ${ }^{o b}$ mutation results in diabetes and an insulin granule stability defect. This present study investigates the functional consequence of the Sorcs1 $\mathrm{Thr}_{52}$ lle mutation in the rat INS1 $\beta$-cell line expressing either the wildtype or mutant Sorcs1 allele. We find that Sorcs1 $\mathrm{Thr}_{52} \mathrm{ll}_{\mathrm{ll}}$ mutation is associated with increased basal insulin secretion, reduced glucose-stimulated insulin secretion and decreased insulin content in INS1 cells. Moreover, expression of Thr $_{52}$ lle causes differential processing of the Sorcs 1 protein resulting in the formation of an additional $90 \mathrm{kDa}$ mutant form of the protein. The mutant form of the protein is localised to the ER, retains its pro-domain, and concurrently reduces expression of the functional mature $130 \mathrm{kDa}$ Sorcs 1 protein. These findings provide a mechanistic clue to why this specific allelic variation in Sorcs1 was associated with reduced insulin levels and type 2 diabetes.

Sorcs1 is a member of the vacuolar protein sorting -10 (VPS10) family of proteins. VPS10, when identified in yeast, was described as a receptor expressed in the trans-Golgi-network (TGN) that trafficks its cargo (the vacuolar hydrolase pro-carboxypeptidase-Y) from the TGN to the endosome ${ }^{1}$. In mammals, there are five members of the VPS10 protein family; Sortilin, SorLA, and Sorcs1-3. Sortilin and SorLA are well-studied and have been shown to play a role in intracellular protein trafficking and sorting, suggesting that all VPS10 proteins may play a similar role.

Sorcs 1 is a type I transmembrane protein that comprises an N-terminal signal peptide, a pro-domain, a Vps10 domain (Vps10-D), a leucine-rich polycystic kidney disease domain, a transmembrane domain, and a short C-terminal cytoplasmic tail ${ }^{2}$, with alternatively spliced isoforms of the protein exhibiting differential subcellular localisation ${ }^{3,4}$. The conserved and characteristic Vps10-D of Sorcs1 and its family members contains a $\beta$-propeller forming a tunnel via which soluble ligands can interact ${ }^{5}$ and be transported between subcellular compartments ${ }^{6}$.

The link between Sorcs 1 mutation and pathophysiology was first implicated in late-onset Alzheimer's disease ${ }^{7}$, in which amyloid precursor protein transport and subsequent amyloid- $\beta$ secretion are affected. Sorcs 1 overexpression correlated with increased amyloid- $\beta$ secretion, while a mutation in Sorcs 1 at the cytoplasmic tail domain increased amyloid- $\beta$ generation in human embryonic kidney cells, demonstrating a specific role for Sorcs 1 in secretory protein retention and anterograde trafficking between the Golgi and the plasma membrane ${ }^{8,9}$.

Using a forward genetics approach employing the diabetes-resistant C57BL/6J (B6) and the diabetes-susceptible Black and Tan Brachyury (BTBR) mouse strains, introgression of the $o b / o b$ allele identified quantitative trait locus for type 2 diabetes on chromosome 19 in the F2 generation ${ }^{10}$. Localisation of this locus in subcongenic BTBR mice expressing both the $o b / o b$ allele and segments of B6 chromosome 19 revealed a single 
nucleotide polymorphism in the Sorcs1 gene (threonine-to-isoleucine substitution at amino acid 52 ( $\mathrm{Thr}_{52} \mathrm{Ile}$ )), associated with the diabetic phenotype ${ }^{10}$. Mice homozygous for the B6 Sorcs1 allele exhibited fasting hyperglycaemia, reduced fasting plasma insulin levels, and irregular pancreatic islet morphology ${ }^{10}$. Subsequently, SORCS1 was identified in several human genome-wide association studies to be associated with diabetes ${ }^{11,12}$ and all of diabetes complications; neuropathy, hypoglycemia unawareness, retinopathy and nephropathy ${ }^{13}$.

In a follow up study, we generated mice with a global deletion of the Sorcs 1 gene. When made obese with the leptin ${ }^{o b}$ mutation, the mice were severely diabetic and the pancreatic $\beta$-cells had a severe deficiency in insulin secretory granules. We concluded that Sorcs 1 is involved in the trafficking and biogenesis of the insulin secretory granules $^{14}$.

While the phenotype of Sorcs 1 knock out mice provided an opportunity to study the consequence of a complete loss-of-Sorcs 1 , it does not necessarily provide true insight into the functional consequences of the non-synonymous $\mathrm{Thr}_{52}$ Ile Sorcs 1 variants associated with type 2 diabetes. The objective of this study was to investigate the consequences of Sorcs $1 \mathrm{Thr}_{52}$ Ile in an in vitro model employing the well-established INS1 $\beta$-cell line. Under stable doxycycline inducible expression of either the wildtype BTBR Sorcs1 allele (wtSorcs1), or the mutant B6 Sorcs1 allele (mutSorcs1), differential processing of the Sorcs1 protein was observed. MutSorcs1-expressing cells demonstrated the formation and endoplasmic reticulum (ER) retention of a $90 \mathrm{kDa}$ mutant pro-protein, which exhibited an extended half life and resulted in reduced total expression of the mature Sorcs 1 protein. Functionally, this reduction was associated with increased basal insulin secretion, blunted glucose-stimulated insulin secretion and reduced total insulin content. As Sorcs1 mutation drives an obesity-regulated diabetic phenotype in animals, these findings have significant implications for the human-associated gene variants and substantiates its role as a genetic contributor to type 2 diabetes.

\section{Materials and Methods}

Cell culture. Glucose-responsive INS1 832/13 (INS1) rat $\beta$-cell line were stably transfected with wild type or mutant Sorcs1 cDNA, with a myc tag at the C-terminal, using the Tet-On system to generate doxycycline-inducible Sorcs1-expressing cells. Cells were cultured in RPMI 1640 medium supplemented with 10\% FBS, 2 mM L-glutamine, $1 \mathrm{mM}$ sodium pyruvate, $50 \mathrm{mM}$ beta-mercaptoethanol, $10 \mathrm{mM}$ HEPES, $1 \%$ penicillin-streptomycin, at $37^{\circ} \mathrm{C}$ in $5 \% \mathrm{CO}_{2}$. Sorcs 1 expression was induced by addition of $2.25 \mu \mathrm{M}$ doxycycline hyclate (Sigma, D9891) into culture media for $18 \mathrm{~h}$ at $37^{\circ} \mathrm{C}$ (or $15^{\circ} \mathrm{C}$ and $20^{\circ} \mathrm{C}$ for temperature trapping experiments).

Generation of doxycycline inducible stable cell lines. The T-REx ${ }^{\mathrm{Tm}}$ system from Invitrogen was used to generate the doxycycline-inducible Sorcs1 wildtype and mutant stable cell lines according to the manufacturer's instructions. Briefly, INS1 832/13 cells, which expresses very low levels of endogenous Sorcs1, were first transfected with pcDNA ${ }^{\mathrm{TM}} 6 / \mathrm{TR}$ plasmid, which encodes the Tet repressor (TetR) under the control of the human CMV promoter, with Lipofectamine 2000 and selected with blasticidin to generate the TetR stable cell lines. A clonal stable cell line that expresses the highest levels of TetR was then used to transfect linearized pCDNA4TOMycHisA-myc-Sorcs1-WT or pCDNA4TOMycHisA-myc-Sorcs1-WT expression vectors and selected with zeocin.

Cell protein extraction and processing. Whole cell lysates (WCL) were obtained from cells using RIPA buffer (Merck, 20-188) containing cOmplete protease inhibitor cocktail (Roche, 4693159001), sonication using a tip probe for $20 \mathrm{~s}$, and centrifugation at $14 \mathrm{k} \mathrm{xg}$ for $10 \mathrm{~min}$. Protein quantification was performed using a BCA protein assay (Pierce, 23225). For disulphide bond reduction, WCL were treated with $10 \mathrm{mM} \mathrm{DTT}$ at $56^{\circ} \mathrm{C}$ for $30 \mathrm{~min}$, then $50 \mathrm{mM} \mathrm{N}$-Ethylmaleimide (NEM) or iodoacetamide (IAA) was added to lysates for $40 \mathrm{~min}$ at RT in the dark prior to SDS-PAGE. For glycan cleavage analysis by western blot, WCL were treated with either $1250 \mathrm{U}$ or 2500 U Endo H (NEB, P0702S) or 500 U PNGase F (NEB, P0709S) under denaturing conditions as per manufacturer's protocols, after overnight induction of Sorcs 1 expression prior to analysis by SDS-PAGE. For proprotein convertase inhibition, cells were incubated in culture media containing $10 \mu \mathrm{M}$ proprotein convertase inhibitor (Calbiochem, 537076) or DMSO control for $30 \mathrm{~min}$ prior to $18 \mathrm{~h}$ induction of Sorcs 1 expression. For protein synthesis inhibition, cells were incubated with either DMSO or $5 \mu \mathrm{M}$ cyclohexamide for up to 2 hours prior to harvest in RIPA buffer.

Western blotting. Cell lysates were analysed on 7.5\% Tris-glycine-SDS-PAGE under reducing conditions of either $0.1 \mathrm{M}$ DTT or $1 \%(\mathrm{v} / \mathrm{v})$ BME, and probed with mouse anti-myc (Merck, 900000032846), mouse anti-beta-actin (Sigma, A5441) and rabbit anti-GAPDH (Santa Cruz, sc-25778) antibodies respectively.

Immunofluorescent staining. Cells were plated onto glass coverslips, fixed with 4\% PFA for 20 min at RT, washed with $0.1 \%$ BSA in PBS with $0.01 \%$ Sodium Azide. Following permeabilisation with $0.1 \%$ SDS for $5 \mathrm{~min}$, cells were blocked with Serum-Free Protein Block (DAKO, X0909) for $1 \mathrm{~h}$ prior to overnight staining at $4^{\circ} \mathrm{C}$ with mouse anti-myc (Merck, 900000032846), guinea pig anti-insulin (DAKO, A0564), rabbit anti-TGN38 (Sigma, T9826), rabbit anti-ERp72 (CST, D70D12) or rabbit anti-Calnexin (Abcam, ab22595) antibodies. Then cells were washed, incubated with fluorescent conjugated secondary antibodies (Invitrogen) for $1 \mathrm{~h}$ at RT in the dark, washed, then mounted onto microscope slides with Prolong Diamond Antifade Mountant containing DAPI (Thermo Fisher, P36965). Slides were imaged using a Leica TCS SP8 confocal microscope using a 100 X oil objective.

Glucose stimulated insulin secretion assay. Cells are plated overnight in culture media, before media is replaced with HEPES Krebs Ringer buffer (KRBH) (20 mM HEPES, pH 7.4; $119 \mathrm{mM} \mathrm{NaCl} ; 4.75 \mathrm{mM} \mathrm{KCl}$;

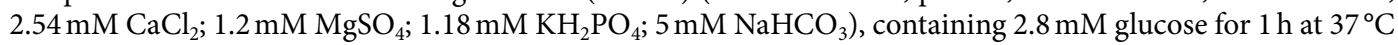
for basal incubation. Cells are then stimulated with $\mathrm{KRBH}$ containing either 2.8 or $16.7 \mathrm{mM}$ glucose for $1 \mathrm{~h}$ at 
$37^{\circ} \mathrm{C}$, and media was collected for insulin secretion determination by Insulin ELISA (Crystal Chem) or Insulin HTRF (Cisbio). Cells were washed then scraped into PBS, centrifuged at $300 \mathrm{xg}$ for $5 \mathrm{~min}$ to pellet, then sonicated for $10 \mathrm{~s}$ in $50 \mathrm{uL}$ of Lysis Buffer $(100 \mathrm{mM}$ Tris, $300 \mathrm{mM} \mathrm{NaCl}, 10 \mathrm{mM} \mathrm{NaF}, 2 \mathrm{mM} \mathrm{Na}$ ) for analysis of total cell DNA concentration (Quant-iT Picogreen DNA kit, Thermo Fisher) and total cell insulin content (HTRF, Cisbio). Insulin secretion and insulin content data are both expressed relative to total cell DNA content.

Subcellular fractionation. Whole cell protein extracts were collected by scraping cells into HEPES/EGTA buffer ( $5 \mathrm{mM}$ HEPES, $1 \mathrm{mM}$ EGTA), lysed by needle pulse by passing 15 times through a 21 -gauge needle, then centrifuged at $1000 \mathrm{xg}, 5 \mathrm{~min}$ and $4^{\circ} \mathrm{C}$. Supernate was layered on the top of a continuous sucrose gradient of $2 \mathrm{M}$ to $0.45 \mathrm{M}$ sucrose in HEPES/EGTA buffer and centrifuged in a Thermo Fisher superspeed centrifuge (A22$24 \times 16$ rotor) for $18 \mathrm{~h}$. Sucrose fractions were collected from the top of the gradient and diluted 2:1 with reducing Laemmli sample buffer (4\% SDS, 20\% glycerol, 10\% beta-mercaptoethanol, $0.004 \%$ bromphenol blue, $0.125 \mathrm{M}$ Tris $\mathrm{HCl}$ ) for analysis by SDS-PAGE.

Sorcs1 immunoprecipitation. WCL was pre-cleared using 50\% Protein G Sepharose beads (GE Life Sciences, 17061801) prior to immunoprecipitation. Precleared WCL was incubated with anti-myc tag antibody-conjugated agarose beads (Merck, 16-219) overnight at $4{ }^{\circ} \mathrm{C}$, centrifuged at $1000 \mathrm{xg}$ for $30 \mathrm{~s}$, washed at least three times with NP-40 buffer, then twice with PBS. Beads were resuspended into 4X Laemmli sample buffer and protein was eluted with incubation at $95^{\circ} \mathrm{C}$ for $10 \mathrm{~min}$ and analysed by SDS-PAGE.

In-gel digestion. Protein eluate was separated in a 7.5\% Tris-glycine SDS-PAGE and incubated with Coomassie brilliant blue G-250 (Sigma, B0770) for $20 \mathrm{~min}$, rinsed in distilled water and destained in 10\% methanol and 7\% acetic acid to remove background staining. Protein bands were excised from the gel, diced into $1 \times 1 \mathrm{~mm}$ pieces and immersed in $30 \%$ acetonitrile and $7 \%$ triethylammonium bicarbonate and incubated in a Thermomixer C (Eppendorf) for $15 \mathrm{~min}$ at $1000 \mathrm{rpm}$ at $22^{\circ} \mathrm{C}$, until gel pieces appeared colourless. The gel was then dried for $5 \mathrm{~min}$ in a GeneVac EZ-2 (SP Scientific), incubated in $100 \mathrm{mM}$ DTT and $100 \mathrm{mM} \mathrm{NH}_{4} \mathrm{HCO}_{3}$ at $56^{\circ} \mathrm{C}$ for $30 \mathrm{~min}$. Supernate was discarded, gel pieces were dehydrated in acetonitrile for $5 \mathrm{~min}$, supernate removed again and gel pieces dried by GeneVac EZ-2 for $5 \mathrm{~min}$ before addition of $200 \mathrm{mM}$ indole-3-acetic acid and $100 \mathrm{mM} \mathrm{NH}_{4} \mathrm{HCO}_{3}$ and incubation at RT for $40 \mathrm{~min}$ in the dark. Supernate was removed, gel pieces dried by GeneVac EZ-2, then rehydrated in $13 \mu \mathrm{g} / \mathrm{ml}$ trypsin in $100 \mathrm{mM} \mathrm{NH}_{4} \mathrm{HCO}_{3}$ at $4^{\circ} \mathrm{C}$ for $1 \mathrm{~h}$, with additional $100 \mathrm{mM}$ $\mathrm{NH}_{4} \mathrm{HCO}_{3}$ added to submerge gel pieces for overnight incubation at $37^{\circ} \mathrm{C}$. Supernate was transferred to a fresh tube, and excess peptides extracted with addition of $60 \%$ acetonitrile $/ 0.1 \%$ trifluoroacetic acid and sonication for $15 \mathrm{~min}$ in a Sonorex Digitec ultrasonic water bath (Bandelin), repeated three times with solution transferred to a fresh tube each time. Final solution was dried using a GeneVac EZ-2.

Peptide desalting. Stage-tips were used in this procedure. Tips were washed once with methanol, once with Elution Buffer (80\% acetonitrile, $0.1 \%$ trifluoroacetic acid) and then three times with Loading Buffer $(0.1 \%$ trifluoroacetic acid). Peptides were resuspended in Loading Buffer and loaded onto the stage-tip, then washed thrice with Loading Buffer. Peptides were eluted in Elution Buffer, then dried using a GeneVac EZ-2.

Liquid chromatography tandem mass spectrometry. Peptides were identified using an UltiMate 3000 (Dionex) paired with a Q-Exactive Plus (Thermo Fisher Scientific) in positive polarity mode. Peptides were separated using an in-house packed $75 \mu \mathrm{m} \times 40 \mathrm{~cm}$ C18AQ column with a $1.9 \mu \mathrm{m}$ particle size. Separation occurred in a $10-35 \% \mathrm{ACN}$ gradient containing $0.1 \%$ Formic acid at $250 \mathrm{nl} / \mathrm{min}$ at $55^{\circ} \mathrm{C}$ for $90 \mathrm{~min}$. MS1 scan was acquired from a $350-1550 \mathrm{~m} / \mathrm{z}$ range with a 70000 resolution, $3 \mathrm{e} 6$ automatic gain control (AGC) and $100 \mathrm{~ms}$ injection time. Data-dependent acquisition of the top 20 ions was achieved using higher-energy collisional dissociation with a 35000 resolution, 1 e6 AGC, $120 \mathrm{~ms}$ injection time, $27 \%$ normalised collision energy and a $1.2 \mathrm{~m} / \mathrm{z}$ isolation width.

MS data analysis. Raw data was processed using MaxQuant (v1.5.3.24) (2) against a hybrid database, including a complete rat Uniprot database (06/2016, 37529 entries) (3), one mouse Sorcs1 protein sequence (Uniprot ID Q9JLC4-2) and the corresponding $\mathrm{Thr}_{52}$ Ile mutant sequence; both containing a C-terminal myc sequence. Processing was conducted using default settings, with the following exceptions: Oxidation of methionine and acetylation of the protein $\mathrm{N}$-terminus were set as variable modifications, and carbaminomethylation of cysteine was set as a fixed modification. Both protein false discovery rate and peptide spectral match were set to $1 \%$. Database-searching was performed twice, with either trypsin or semispecific trypsin as the digestion mode; combining the identification results.

Statistics. All statistical analysis was performed in GraphPad Prism version 8.2.0. Statistical significance was determined by two-tailed student's t-test or one-way ANOVA with Tukey's multiple comparisons post-test. A value of $p<0.05$ was considered significant.

\section{Results}

Sorcs1 Thr52lle mutation results in defective basal insulin secretion. It was a $\mathrm{Thr}_{52}$ Ile mutation in the pro-domain of the mouse Sorcs 1 protein that led to the identification of the gene as a type 2 diabetes gene ${ }^{10}$ (Fig. 1A). We will refer to this allele as mutSorcs 1 . This mutation causes a reduction in insulin levels and congenic mice with insertion of the B6 mutSorcs 1 allele in the BTBR background display lower fasting plasma insulin as well as a reduction in insulin during a glucose tolerance test validating that mutSorcs 1 underlies the associated phenotype ${ }^{10}$. In this study, we assesed the functional consequence of mutSorcs 1 in vitro by expressing the two variants in INS-1 $\beta$-cells and measured glucose-stimulated insulin secretion and insulin content. MutSorcs 1 
A

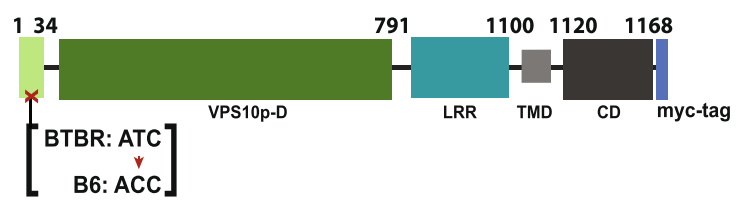

B

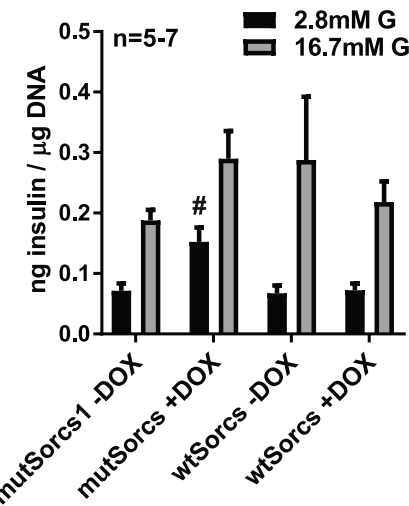

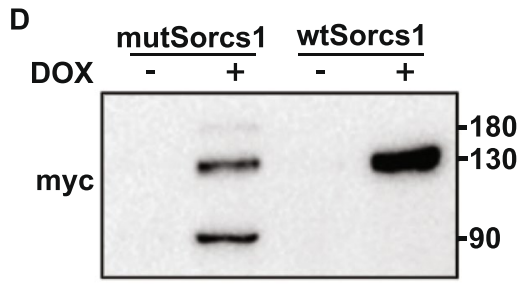

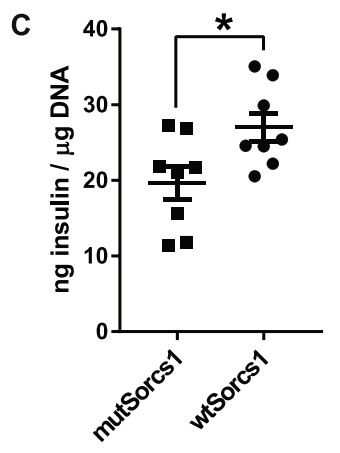

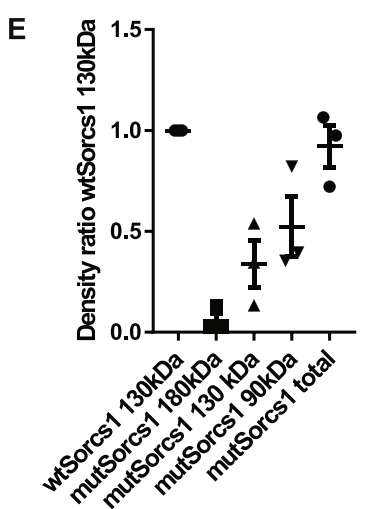

$\mathbf{F}$

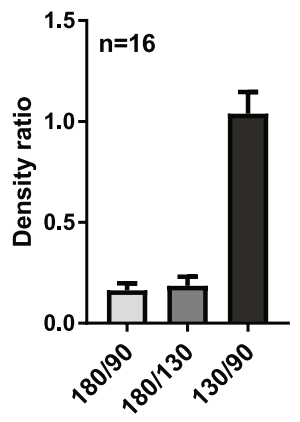

Figure 1. Alternate protein products in Sorcs1 $\mathrm{Thr}_{52} \mathrm{Ile}$ SNP result in defective insulin secretion. (A) Graphical representation of Sorcs1 protein and single-nucleotide polymorphism in pro-domain region. (B) Glucoseinduced insulin secretion assay performed in wtSorcs1 and mutSorcs1-expressing INS1 cells. Cells were incubated in basal media of $2.8 \mathrm{mM}$ glucose for $1 \mathrm{~h}$, then stimulated at $2.8 \mathrm{mM}$ or $16.7 \mathrm{mM}$ glucose for $1 \mathrm{~h}$. Secreted insulin in media was measured by HTRF assay, then expressed relative to total cellular DNA content. (C) Insulin content measured by HTRF normalised to total cellular DNA content of wtSorcs1 and mutSorcs 1 expressing INS cells after glucose-stimulated insulin secretion assay. (D) Representative western blot of lysates from $w t$ Sorcs 1 and $m u t S o r c s 1$-expressing INS1 cells, harvested after $18 \mathrm{~h}$ doxycycline (or DMSO control) treatment. Cells were scraped into RIPA buffer containing protease inhibitors, sonicated, then centrifuged to collect lysates. Lysates were run on $7.5 \%$ tris-glycine gels for western blotting. (E) Densitometry analysis of alternative products observed in myc immunoblot bands in mutSorcs 1 -expressing INS1 cells. (F) Densitometry analysis comparing alternate mutSorcs 1 INS bands and $w t$ Sorcs 1 bands in myc immunoblot. *p $<0.05$ by oneway ANOVA, Tukey's multiple comparison post-test, ${ }^{*} \mathrm{p}<0.05$ versus all basal groups by one-way ANOVA, Tukey's multiple comparison post-test.

expressing INS-1 cells exhibited significantly heightened basal levels of insulin secretion, with an approximate doubling of insulin secretion under basal conditions ( $p<0.05$ by one-way ANOVA), and reduced glucose-stimulated insulin secretion (Fig. 1B). Further, cellular insulin content of mutSorcs1 expressing INS-1 cells was significantly lower compared to cells expressing wtSorcs1 (Fig. 1C), validating that our cell system phenocopies the loss of insulin associated with the mutation in mice.

Sorcs 1 Thr52lle mutation forms an uncleaved protein product of $90 \mathrm{kDa}$. Sorcs 1 is translated as a precursor protein (pro-Sorcs1) which is processes to its mature $130 \mathrm{kDa}$ form by furin-mediated cleavage of an $\mathrm{N}$-terminal 77 animo acids pro-peptide ${ }^{15}$. We obtained a clue to the functional consequence of $m u t$ Sorcs 1 when we expressed the two variants that contained C-terminal myc-tag in INS1 $\beta$-cells, under a doxycycline-inducible system. On 7.5\% SDS-PAGE, immunoblotting of the myc-tag showed that $w t$ Sorcs 1 allele expression results in the formation of a large band at $130 \mathrm{kDa}$ corresponding to the mature protein. However, expression of the $\mathrm{Thr}_{52} \mathrm{Ile}$ mutSorcs 1 allele was visualised as three unique bands corresponding to not only the $130 \mathrm{kDa}$ mature protein band, but additional bands at approximately $90 \mathrm{kDa}$ and $180 \mathrm{kDa}$ (Fig. 1D). It was likely that the large band observed at $130 \mathrm{kDa}$ also contained the pro-form of the Sorcs 1 (pro-Sorcs1), and the mature $130 \mathrm{kDa}$ band of both $w t S o r c s 1$ and $m u t$ Sorcs 1 resolved as a doublet containing pro-Sorcs1 and mature Sorcs1 in an 7.5\% SDS-PAGE when run for an extended duration to specifically separate out higher density proteins (Supplementary Fig. 1A). This was also observed when Sorcs1 was separated on a Bolt ${ }^{\mathrm{TM}} 4-12 \%$ Bis-Tris Plus Gels in Bolt MOPS SDS running buffer (Supplementary Fig. 1B). Densitometry analysis of the mutSorcs 1 bands demonstrated that their cumulative density was similar to that of the $130 \mathrm{kDa} w t$ Sorcs 1 band (Fig. 1E), while densitometry analysis of multiple mutSorcs 1 samples indicated that the $90 \mathrm{kDa}$ and $130 \mathrm{kDa}$ bands constituted the predominant forms of the protein (Fig. 1F). We hypothesised that a processing defect, such as additional $\mathrm{N}$-terminal cleavage of mutSorcs1 (as both forms possess the c-terminal myc-tag), could be responsible for the lighter protein product.

Additional $90 \mathrm{kDa}$ mutSorcs 1 band is not altered by proprotein cleavage. Conversion of pro-Sorcs1 to its mature form normally occurs through processing by furin-mediated cleavage at two sites ${ }^{15}$, however, an additional pro-protein convertase cleavage site was predicted to exist in both Sorcs 1 alleles $^{16}$. As the $90 \mathrm{kDa}$ mutSorcs 1 band retained its C-terminal myc tag, we aimed to investigate if the mutSorcs 1 protein 
was the result of additional cleavage sites. To investigate this, both $w t$ Sorcs 1 and mutSorcs 1 - expressing INS-1 cells were treated with a cell permeable pro-protein convertase inhibitor (PCI) targeted to furin, but also PC1/3, PC4, PC5/6 and PACE $4{ }^{17}$ in a dose curve (Supplementary Fig. 2A). SDS-PAGE of control and PCI treated cells revealed a small shift in the $130 \mathrm{kDa}$ band in both wtSorcs1 and mutSorcs1-expressing cells to a slightly heavier product at a $10 \mu \mathrm{M}$ treatment dose, corresponding to a density shift to the pro-Sorcs1 protein (Fig. 2A). A specific furin-inhibitor also mediated the same shift in the $130 \mathrm{kDa}$ wtSorcs 1 band (Supplementary Fig. 2B). However, no change in mass was observed in either the lighter $90 \mathrm{kDa}$ fragment or the heavier $180 \mathrm{kDa}$ fragment of Sorcs 1 resulting from expression of the mutSorcs 1 allele, indicating that its generation was unaffected by pro-protein convertase activity.

To more definitively characterise the mutSorcs $190 \mathrm{kDa}$ product, peptide identification by LC-MS/MS was performed on $130 \mathrm{kDa}$ and $90 \mathrm{kDa}$ protein bands excised from gels processed from immunoprecipitated Sorcs 1 in $w$ tSorcs 1 and mutSorcs 1 - expressing INS1 samples (Fig. 2B). As expected, the $130 \mathrm{kDa}$ band (corresponding to both pro-Sorcs 1 and mature Sorcs 1 ) products from wtSorcs 1 and mutSorcs 1 demonstrated similar peptide coverage. Most strikingly, peptide coverage of the mutSorcs $190 \mathrm{kDa}$ product also spanned coverage of the pro-domain of the Sorcs1 protein (Fig. 2C). In agreement with the PCI experiments, these data demonstrate that the protein size discrepancy is not a result of $\mathrm{N}$-terminal cleavage of the mutant protein. Raw peptide coverage data for all three experiments are included in Supplementary Table 1, and peptide quantification by trypsin and semi-specific trypsin digestion excluded peptide contamination.

Additional $90 \mathrm{kDa}$ mutSorcs 1 band is not altered by disulphide bonds. All available cysteines within the common Vps10p-D of Sortilin, including 10 of the conserved cysteines within the C-terminal region, have been shown to form disulphide bonds ${ }^{18}$. Using prediction software analysis, Sorcs 1 is expected to have 10 high confidence disulphide bonds. Therefore, we tested the possibility that altered intermolecular disulphide bonding was the cause of the additional mutSorcs 1 protein bands observed. INS1 cells expressing wtSorcs 1 and mutSorcs 1 were immunoblotted in both reducing and non-reducing conditions, however the migration of all mutSorcs1 protein bands were unchanged (Fig. 2D). Densitometry analysis also found no difference between density ratios of the $130 \mathrm{kDa}$ and $90 \mathrm{kDa}$ mutSorcs 1 bands in reducing and non-reducing conditions (Fig. 2E).

As the reducing agents used above (DTT and 2-mercaptoethanol) were potentially reversible, we validated these findings by performing irreversible blocking of cysteine oxidation with iodoacetamide or NEM under reducing conditions. A small shift in motility of all mutSorcs 1 bands, as well as the $130 \mathrm{kDa} w t$ Sorcs 1 band (Fig. 2F) indicated that all protein products contained oxidized cysteines - though their presence in the $90 \mathrm{kDa}$ mutSorcs 1 was not enough to account for the molecular mass discrepancy compared to $w t$ Sorcs 1 . To eliminate potential misinterpretation of data due to reagents diffusing between adjacent lanes during SDS-PAGE, we included blank lanes between reduced versus non-reduced samples and obtained similar results (Supplementary Fig. 2C).

The $90 \mathrm{kDa}$ mutSorcs1 protein product is formed and retained in the ER. Sequence analysis of murine Sorcs 1 predicted the presence of eight $N$-linked glycans ${ }^{19}$. As such, alternative post-translational glycosylation in mutSorcs 1 was investigated as the cause of the $90 \mathrm{kDa}$ product. INS1 cells expressing $w t$ Sorcs 1 and mutSorcs 1 were subjected to glycan cleavage by either Endoglycosidase $\mathrm{H}$ (Endo $\mathrm{H}$ ) - which specifically cleaves high-mannose $N$-linked glycans, or Peptide- $N$-Glycosidase F (PNGase F) - which cleaves almost all $N$-linked glycans. Each deglycosylation reaction demonstrated distinct mobility shifts for pro-Sorcs1 and mature Sorcs 1 bands in both wtSorcs 1 and mutSorcs 1 lysates. As expected, pro-Sorcs1 (red arrows) - but not processed mature Sorcs1 (black arrows) - shifted to a lower molecular weight when exposed to Endo H, consistent with its localisation within the ER as a uncleaved pro-peptide (Fig. 3A). PNGase F decreased the mass of pro-Sorcs1, and also mature Sorcs 1, corresponding with the increasingly de-glycosylated Sorcs1 protein (Fig. 3B). Of significant interest is that the band shifts apparent in both the pro-Sorcs 1 protein and $90 \mathrm{kDa}$ mutSorcs 1 product were identical in both Endo $\mathrm{H}$ treatment and PNGase F treatment (red and blue arrows, Fig. 3C), indicating the presence of only high-mannose $\mathrm{N}$-linked glycans in these proteins - and a lack of Endo $\mathrm{H}$ resistance. This data suggests that, like pro-Sorcs1, the $90 \mathrm{kDa}$ mutSorcs 1 protein is ER localised.

To investigate this further we performed subcellular fractionation analysis of INS-1 cells expressing either the $w t$ Sorcs 1 or mutSorcs 1 allele to identify potential changes in subcellular localisation (Fig. 3D). Total densities of $w t$ Sorcs 1 and mutSorcs 1 bands co-fractionated almost identically (Fig. 3E), however, comparison of the two mutSorcs 1 bands identified subtle differences in subcellular localisation between the $130 \mathrm{kDa} w t$ Sorcs 1 -corresponding band and the $90 \mathrm{kDa}$ band, with peak densities of the latter appearing in heavier sucrose fractions (Fig. 3F).

To further investigate Sorcs1 subcellular distribution, immunofluorescent staining of myc and insulin was performed in both myc-tagged $w t$ Sorcs 1 and mutSorcs 1 expressing INS1 cells. Myc was initially co-stained with Sorcs1 to confirm significant co-localisation, with both appearing in reticular structures in the cell periphery (Supplementary Fig. 3A). Insulin co-staining with myc demonstrated no obvious co-localisation between Sorcs1 and insulin granules, in either $w t$ Sorcs 1 or mutSorcs 1 (Supplementary Fig. 3B), though insulin granules appeared characteristically punctate in the cell periphery, with increased intensity in perinuclear regions likely corresponding to pro-insulin (or immature insulin granules) in the TGN. As we previously reported ${ }^{14}$, myc/Sorcs 1 demonstrated co-localisation with the TGN marker TGN38, notably here in both wtSorcs 1 and mutSorcs 1 INS1 cells (Fig. 4A), however only mutSorcs 1 INS1 cells displayed consistent partial co-localisation with the ER markers ERp72 or Calnexin (Fig. 4B,C). Quantification by Pearsons correlation coefficient demonstrated significantly increased co-localisation of ER markers in mutSorcs 1 -expressing cells than $w$ tSorcs 1 -expressing cells, but no difference in TGN38 co-localisation (Fig. 4D, p < 0.01). 
A

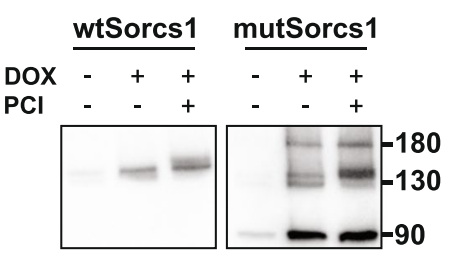

B

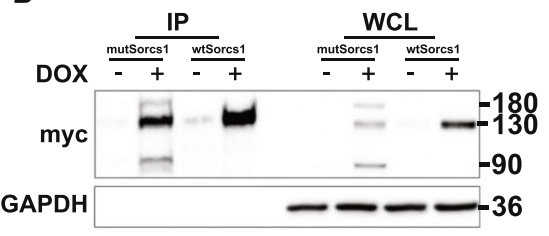

C

mutSorcs1: 90kDa

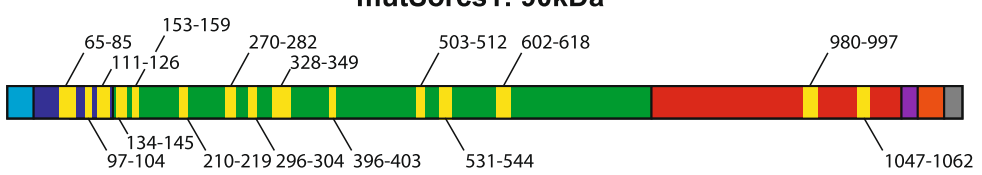

Signal peptide

Pro-domain

Vps10 domain

mutSorcs1: $130 \mathrm{kDa}$

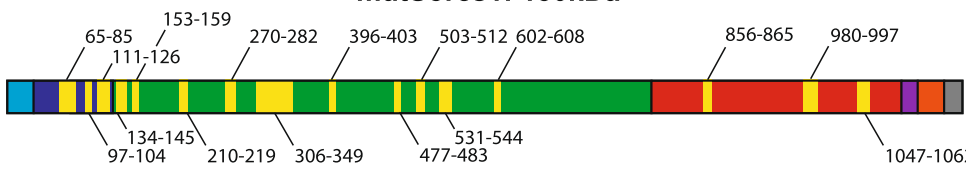

Leucine rich

region

Transmembrane

domain

Cytoplasmic

tail

wtSorcs 1: 130kDa

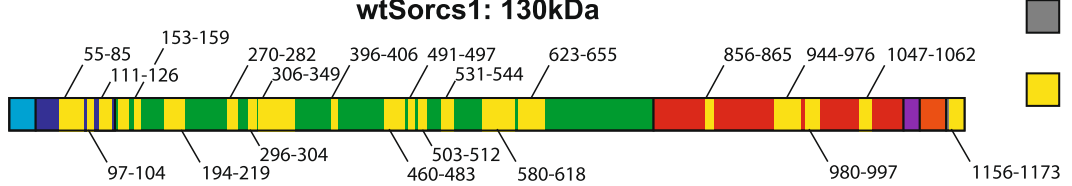

Myc tag

MS/MS identified region

D

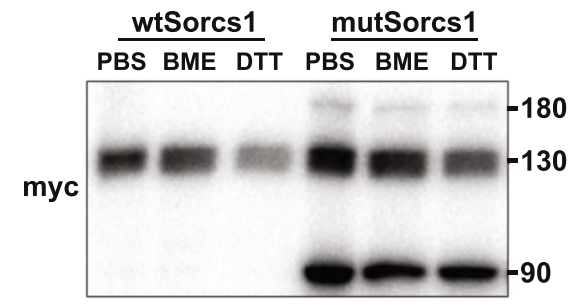

E

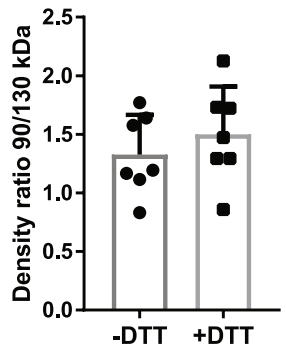

F

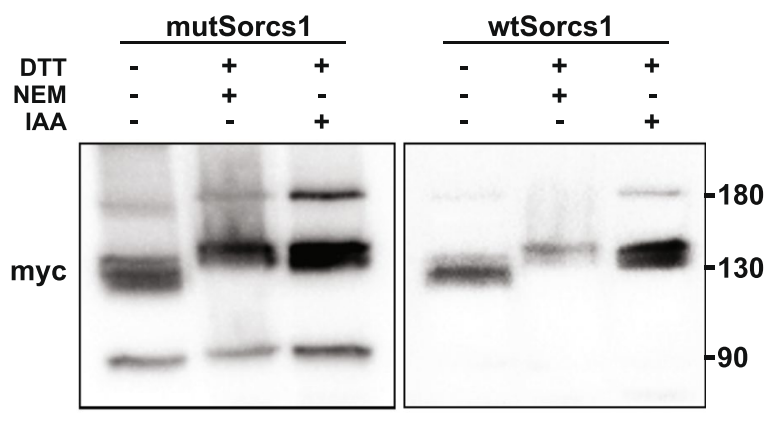

Figure 2. The alternative $90 \mathrm{kDa}$ protein product of mutSorcs 1 is unaffected by pro-protein convertases. (A) Representative western blot of lysates from $w t$ Sorcs 1 and $m u t$ Sorcs 1-expressing INS1 cells treated with DMSO or $10 \mu \mathrm{M}$ prohormone convertase inhibitor (PCI). (B) Immunoprecipitation of Sorcs 1 from $w t$ Sorcs 1 and mutSorcs1-expressing INS1 whole cell lysates (WCL) using anti-myc tag antibody-conjugated agarose beads. (C) Representative peptide coverage of $130 \mathrm{kDa}$ and $90 \mathrm{kDa}$ bands excised from SDS-PAGE of wtSorcs 1 and mutSorcs1-expressing INS1 cells and subject to LC-MS/MS data analysis. (D) Representative western blot of $w t$ Sorcs 1 and mutSorcs 1 -expressing INS1 cells in reducing and non-reducing conditions. (E) Densitometry analysis of mutSorcs 1 bands immunoblotted in the presence or absence of $100 \mathrm{mM}$ dithiothreitol (DTT). (F) Representative western blot of $w t$ Sorcs 1 and mutSorcs 1 -expressing INS1 cells in reducing conditions in the presence of $50 \mathrm{mM}$ N-Ethylmaleimide (NEM) or iodoacetamide (IAA). 
A

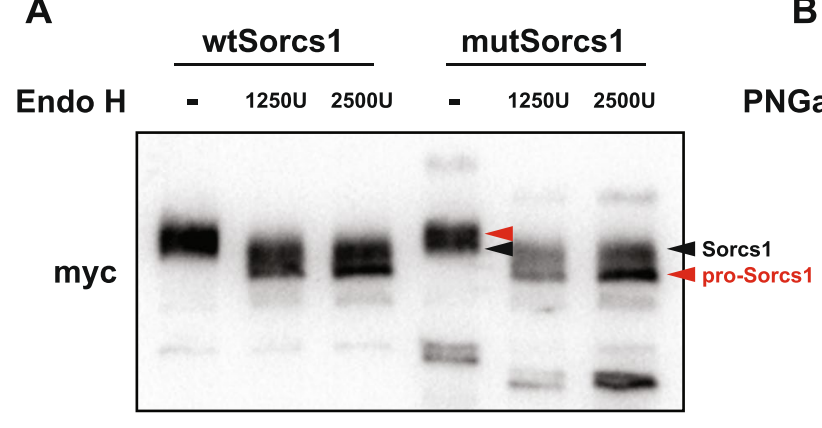

C

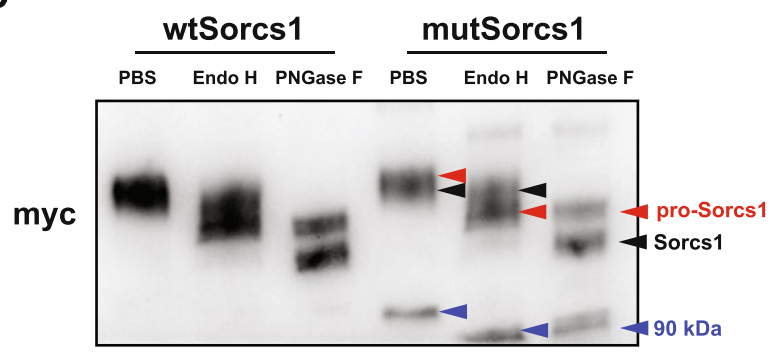

B wtSorcs1 mutSorcs1

D

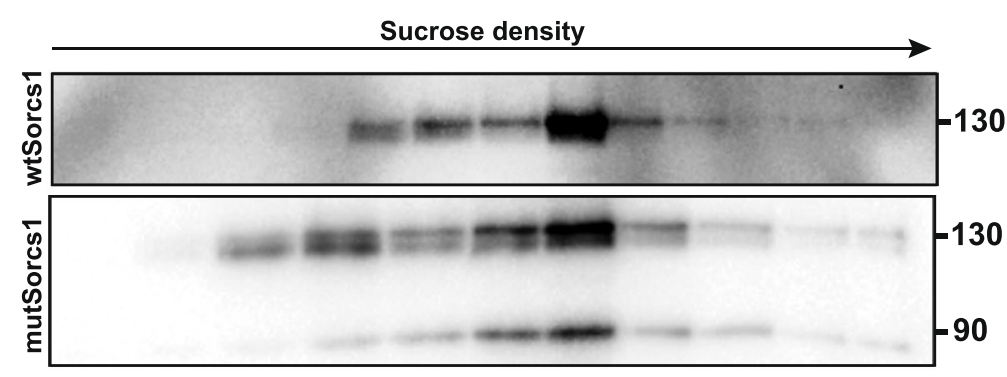

E

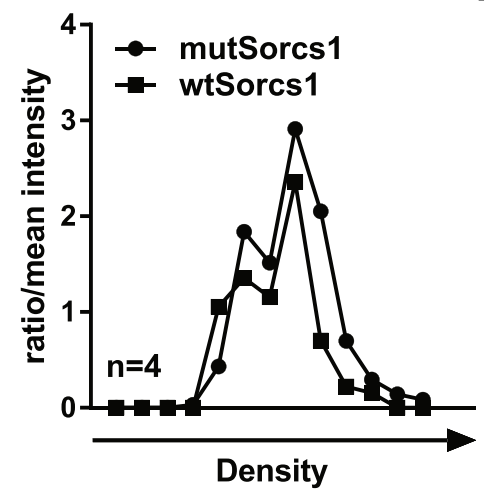

$\mathbf{F}$

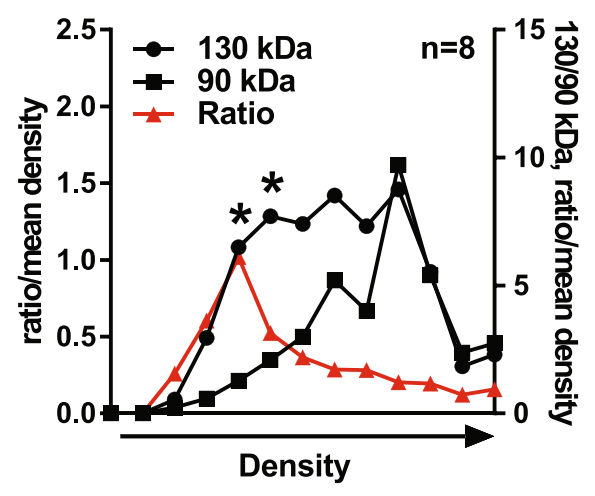

Figure 3. The alternative $90 \mathrm{kDa}$ protein product exhibits a different glycosylation profile. (A) Representative western blot of $w t$ Sorcs 1 and mutSorcs 1 INS1 cells after de-glycosylation treatment with $1250 \mathrm{U}$ or $2500 \mathrm{U}$ Endo H. (B) Representative western blot of wtSorcs 1 and mutSorcs1 INS1 cells after de-glycosylation treatment with 500 U PNGase F. (C). Western blot of $w t$ Sorcs 1 and mutSorcs1 INS1 cells after de-glycosylation treatment with 1250 U Endo H and 500 U PNGase F. (D) Representative western blot of subcellular fractionation by sucrose gradient of wtSorcs1 and mutSorcs 1-expressing INS1 cells. (E) Densitometry analysis of combined bands in subcellular fractionation immunoblots in wtSorcs 1 and mutSorcs1 INS1 cells. (F) Densitometry analysis of 180, 130 and $90 \mathrm{kDa}$ mutSorcs 1 INS1 cell bands in subcellular fractionation immunoblots. $* \mathrm{p}<0.05$, unpaired t-test of ratio/mean density of $130 \mathrm{kDa}$ compared to $90 \mathrm{kDa}$ band in Fraction 5 and Fraction 6

MutSorcs1 90 kDa exhibits a different half life to the wildtype protein. As these data lend evidence to the presence of a differentially modified, ER-localised mutant Sorcs 1 product, we treated $w t$ Sorcs 1 and mutSorcs1-expressing INS1 cells with cyclohexamide to investigate protein degradation. We found that 
A

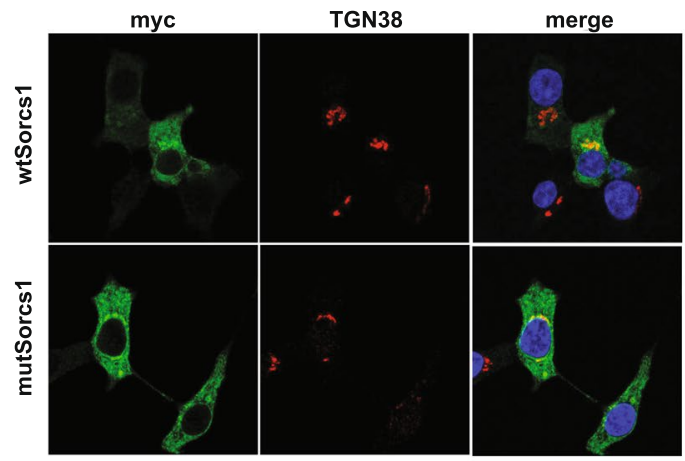

B

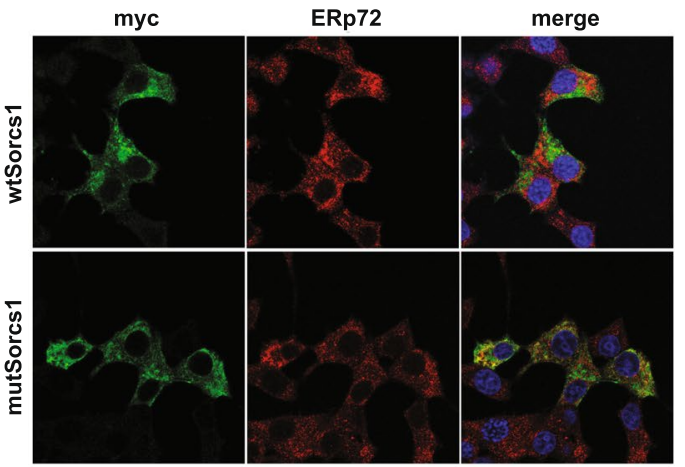

C

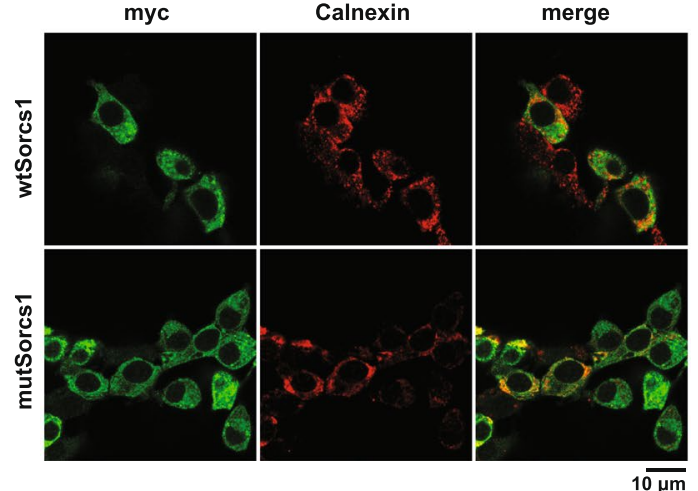

D

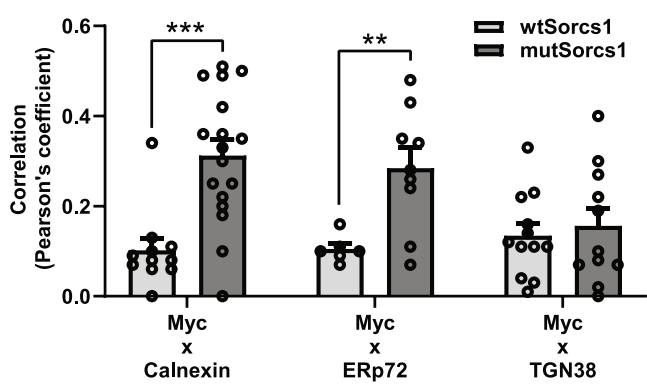

Figure 4. The alternative $90 \mathrm{kDa}$ protein product has different localisation to the mature protein. (A) Representative confocal images from immunofluorescent staining of myc and TGN38 in wtSorcs 1 and mutSorcs 1-expressing INS1 cells. (B) Representative confocal images from immunofluorescent staining of myc and ERp72 in wtSorcs 1 and mutSorcs1-expressing INS1 cells. (C) Representative confocal images from immunofluorescent staining of myc and Calnexin in wtSorcs 1 and mutSorcs 1 -expressing INS1 cells. Representative images were selected from 6-10 Z-stack images, containing 1-4 cells per image, across two separate experiments. (D) Peasons coefficient colocalisation analysis of myc and Calnexin, myc and ERp72 and myc and TGN38 in wtSorcs 1 and mutSorcs 1- expressing cells averaged from 6-17 images across two separate experiments. $* * * \mathrm{p}<0.001, * * \mathrm{p}<0.01$ by unpaired $\mathrm{t}$-test. 
A

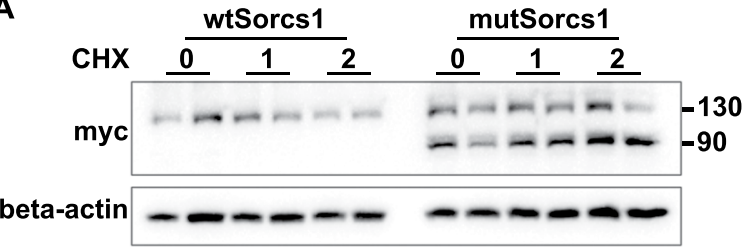

B

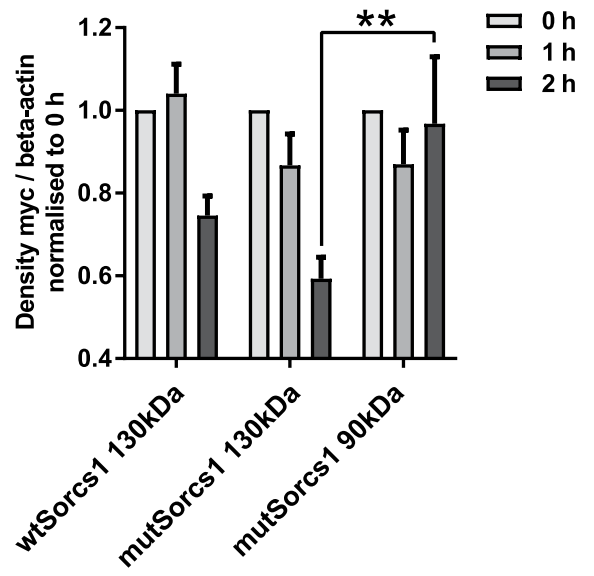

Figure 5. The alternative $90 \mathrm{kDa}$ protein product has an increased half-life. (A) Representative western blot of $w t$ Sorcs 1 and mutSorcs 1 -expressing cells after DMSO or $5 \mu \mathrm{M}$ cyclohexamide (CHX) treatment for 1 and 2 hours. (D) Densitometry analysis of $130 \mathrm{kDa}$ and $90 \mathrm{kDa}$ bands in cyclohexamide treatment of $w t \operatorname{Sorcs} 1$ and mutSorcs 1 -expressing cells. **p $<0.01$ by one-way ANOVA, Tukey's multiple comparison post-test.

mutSorcs 1 -expressing cells demonstrated increased retention of the mutant $90 \mathrm{kDa}$ protein 2 hours post-treatment compared to the $130 \mathrm{kDa}$ protein product $(\mathrm{p}<0.01$, Fig. $5 \mathrm{~A}, \mathrm{~B})$, further validating an alternate processing pathway for the mutant form protein associated with the $\mathrm{Thr}_{52}$ Ile single nucleotide polymophism in Sorcs1.

\section{Discussion}

Natural genetic variations can offer better mechanistic insights into complex biological processes that are more informative than investigations using complete gene deletion. This is because a mutation in a gene can either be a loss or gain-of-function mutation. The objective of this study was to elucidate the functional consequence of the $\mathrm{Thr}_{52}$ Ile single nucleotide polymorphism in the pro-domain of the Sorcs 1 gene that has been associated with type 2 diabetes. Expression of the mutant allele of Sorcs 1 in INS1 cells clearly demonstrated alternative processing of the Sorcs 1 protein compared to wildtype Sorcs1 when visualised by SDS-PAGE. The significantly lower molecular mass of the mutant protein was striking. The formation of an additional protein band of $90 \mathrm{kDa}$ initially suggested that an additional cleavage of mutSorcs 1 by a protease such as furin, however pro-protein convertase inhibition resulted in no change in the size of the alternative band. Furthermore, an upward band shift of the mature $130 \mathrm{kDa}$ band indicated that there was normally efficient processing of the mature Sorcs 1 protein from the pro-form in both mutSorcs 1 and $w t$ Sorcs 1 - expressing INS1 cells.

It appears that the $90 \mathrm{kDa}$ product of mutSorcs 1 protein is misfolded, though we have been unable to definitively confirm this in our current study. Despite at least 10 cysteine sites at which intramolecular disulphide bonds were predicted to occur, irreversible blocking of cysteine oxidation eliminated a role for disulphide bonds as a source for alternatively folded mutSorcs 1 protein products. The retention of the pro-domain as identified by LC-MS/MS, and high-mannose glycosylation profile indicates that this $90 \mathrm{kDa}$ product of mutSorcs 1 protein does not completely access the TGN - in which furin-mediated pro-domain cleavage, and hybrid or complex glycosylation occurs. Subcellular fractionation of INS1 further supports the notion that the additional mutSorcs 1 protein band localises differently to the $130 \mathrm{kDa} w t$ Sorcs 1 protein band and therefore undergoes differential processing through subcellular compartments. Immunofluorescent staining also demonstrated ERp72, calnexin and myc co-staining primarily in mutSorcs 1 INS1 cells and not in wtSorcs1 INS1 cells, with TGN38 co-localisation in both cell lines. Finally, the $90 \mathrm{kDa}$ mutSorcs1 protein exhibits an increased half life compared to the $130 \mathrm{kDa}$ protein, providing further evidence for its mis-localisation and abnormal trafficking within the cell.

From a functional perspective, earlier work with congenic mouse strains, we found that mutSorcs 1 allele in the BTBR mouse background showed impaired insulin secretion in response to an intraperitonial glucose challenge as well as a $30 \%$ reduction in fasting plasma insulin relative to the BTBR mouse ${ }^{10}$. We hypothesize that one explanation for this observation is that insertion of mutSorcs1 into the BTBR mouse strain background favors the formation of the misfolded Sorcs1 protein to a greater extent than we observed in the INS1 $\beta$-cell line.

Our data suggests that the alternatively processed or misfolded mutSorcs 1 products accumulate in the ER and reduce the expression of mature $130 \mathrm{kDa}$ Sorcs 1 protein which is a major determinant of insulin content. This reduction in mature $130 \mathrm{kDa}$ Sorcs 1 , reduces normal $\beta$-cell function. The increased insulin secretion at $2.8 \mathrm{mM}$ 
glucose observed in mutSorcs1 INS1 cells is also reminiscent of the elevated basal (pro)insulin secretion characteristic of Type 2 Diabetes, and indicative of dysregulated proximal insulin processing. Though these data are incongruent with the reduced plasma insulin levels seen in B6 Sorcs 1 allele-expressing mice ${ }^{10}$, it is likely that the elimination of whole-body Sorcs1 is incomparable with the more subtle phenotype of a single nucleotide polymorphism. Pro-peptide cleavage in the TGN of VPS10 proteins is required for exposure of ligand-binding regions in both Sortilin ${ }^{20}$ and SorLA, and these pro-peptides are then capable of binding to its own mature protein ${ }^{18}$. This is hypothesised to be a form of self-regulation by either assisting with protein-folding or to inhibit premature ligand binding ${ }^{18}$. While Sorcs1 pro-peptide has low affinity for mature Sorcs1, it instead is capable of binding mature Sortilin ${ }^{15}$. Sorcs 1 has thereby been implicated in post-Golgi cargo trafficking, primarily through its inhibition of Sortilin-regulated ligand uptake and transcription factor interaction by competitive binding of mature Sortilin protein ${ }^{15}$. As such, it stands to reason that a $50 \%$ reduction of the mature $130 \mathrm{kDa}$ Sorcs 1 protein may in turn result in an increase of Sortilin function. This is further evidenced in INS1 cells overexpressing Sortilin, which exhibit decreased total insulin content (unpublished observations), implicating a role for Sortilin in the downregulation of insulin within the $\beta$-cell, potentially through its role in lysosomal trafficking ${ }^{21,22}$. However, although Sortilin is a known receptor for soluble ligands such as amyloid precursor protein ${ }^{23}$, neurotensin ${ }^{24}$, PCSK9 $^{25}$, as well as being involved in retrograde trafficking of GLUT4 ${ }^{26}$, it is not yet known whether sortilin is also capable of regulating proinsulin trafficking.

Polymorphisms affecting Sorcs1 function have previously been reported ${ }^{7,12,27}$. Similarly, pro-domain missense mutations are not uncommonly associated with disease ${ }^{28,29}$. We have demonstrated that the $\mathrm{Thr}_{52}$ Ile mutant Sorcs1 product is subject to impaired processing in the $\beta$-cell. Sorcs 1 trafficking and function in particular, appears highly regulated by its pro-domain. We speculate that aberrant function of $\beta$-cells associated with $\mathrm{Thr}_{52} \mathrm{Ile}_{\mathrm{I}}$ mutation in the Sorcs 1 gene is driven by the stoichiometric loss of wildtype Sorcs 1 protein in favour of the mutant form of the protein. This, in turn, may imbalance the trafficking network associated with normal insulin content and secretory function.

\section{Data availability}

The datasets generated during and/or analysed during the current study are available from the corresponding author on reasonable request.

Received: 13 September 2019; Accepted: 3 December 2019;

Published online: 19 December 2019

\section{References}

1. Marcusson, E. G., Horazdovsky, B. F., Cereghino, J. L., Gharakhanian, E. \& Emr, S. D. The sorting receptor for yeast vacuolar carboxypeptidase $\mathrm{Y}$ is encoded by the VPS10 gene. Cell 77, 579-586 (1994).

2. Savas, J. N. et al. The Sorting Receptor SorCS1 Regulates Trafficking of Neurexin and AMPA Receptors. Neuron 87, 764-780, https:// doi.org/10.1016/j.neuron.2015.08.007 (2015).

3. Hermey, G. et al. Characterization of sorCS1, an alternatively spliced receptor with completely different cytoplasmic domains that mediate different trafficking in cells. Journal of Biological Chemistry 278, 7390-7396, https://doi.org/10.1074/jbc.M210851200 (2003).

4. Nielsen, M. S. et al. Different motifs regulate trafficking of SorCS1 isoforms. Traffic 9, 980-994, https://doi.org/10.1111/j.16000854.2008.00731.x (2008).

5. Horazdovsky, B. F., DeWald, D. B. \& Emr, S. D. Protein transport to the yeast vacuole. Curr Opin Cell Biol 7, 544-551 (1995).

6. Deloche, O., Yeung, B. G., Payne, G. S. \& Schekman, R. Vps10p transport from the trans-Golgi network to the endosome is mediated by clathrin-coated vesicles. Molecular biology of the cell 12, 475-485, https://doi.org/10.1091/mbc.12.2.475 (2001).

7. Reitz, C. et al. SORCS1 alters amyloid precursor protein processing and variants may increase Alzheimer's disease risk. Ann Neurol 69, 47-64, https://doi.org/10.1002/ana.22308 (2011).

8. Lane, R. F. et al. Protein sorting motifs in the cytoplasmic tail of SorCS1 control generation of Alzheimer's amyloid-beta peptide. $J$ Neurosci 33, 7099-7107, https://doi.org/10.1523/JNEUROSCI.5270-12.2013 (2013).

9. Lane, R. F. et al. Diabetes-associated SorCS1 regulates Alzheimer's amyloid-beta metabolism: evidence for involvement of SorL1 and the retromer complex. J Neurosci 30, 13110-13115, https://doi.org/10.1523/JNEUROSCI.3872-10.2010 (2010).

10. Clee, S. M. et al. Positional cloning of Sorcs1, a type 2 diabetes quantitative trait locus. Nat Genet 38, 688-693, https://doi. org/10.1038/ng1796 (2006).

11. Goodarzi, M. O. et al. SORCS1: a novel human type 2 diabetes susceptibility gene suggested by the mouse. Diabetes 56, 1922-1929, https://doi.org/10.2337/db06-1677 (2007).

12. Florez, J. C. et al. A $100 \mathrm{~K}$ genome-wide association scan for diabetes and related traits in the Framingham Heart Study: replication and integration with other genome-wide datasets. Diabetes 56, 3063-3074, https://doi.org/10.2337/db07-0451 (2007).

13. Paterson, A. D. et al. A genome-wide association study identifies a novel major locus for glycemic control in type 1 diabetes, as measured by both A1C and glucose. Diabetes 59, 539-549, https://doi.org/10.2337/db09-0653 (2010).

14. Kebede, M. A. et al. SORCS1 is necessary for normal insulin secretory granule biogenesis in metabolically stressed beta cells. J Clin Invest 124, 4240-4256, https://doi.org/10.1172/JCI74072 (2014).

15. Larsen, J. V. et al. Human sorCS1 binds sortilin and hampers its cellular functions. Biochem J 457, 277-288, https://doi.org/10.1042/ BJ20130386 (2014).

16. Duckert, P., Brunak, S. \& Blom, N. Prediction of proprotein convertase cleavage sites. Protein Eng Des Sel 17, 107-112, https://doi. org/10.1093/protein/gzh013 (2004).

17. Becker, G. L. et al. Highly potent inhibitors of proprotein convertase furin as potential drugs for treatment of infectious diseases. $J$ Biol Chem 287, 21992-22003, https://doi.org/10.1074/jbc.M111.332643 (2012).

18. Westergaard, U. B. et al. Functional organization of the sortilin Vps10p domain. J Biol Chem 279, 50221-50229, https://doi. org/10.1074/jbc.M408873200 (2004).

19. UniProt, C. UniProt: a hub for protein information. Nucleic Acids Res 43, D204-212, https://doi.org/10.1093/nar/gku989 (2015).

20. Munck Petersen, C. et al. Propeptide cleavage conditions sortilin/neurotensin receptor-3 for ligand binding. Embo $j$ 18, 595-604, https://doi.org/10.1093/emboj/18.3.595 (1999).

21. Tanimoto, R. et al. Sortilin regulates progranulin action in castration-resistant prostate cancer cells. Endocrinology 156, 58-70, https://doi.org/10.1210/en.2014-1590 (2015).

22. Zhou, X., Sullivan, P. M., Paushter, D. H. \& Hu, F. The Interaction Between Progranulin with Sortilin and the Lysosome. Methods in molecular biology (Clifton, N.J.) 1806, 269-288, https://doi.org/10.1007/978-1-4939-8559-3_18 (2018). 
23. Gustafsen, C. et al. Sortilin and SorLA display distinct roles in processing and trafficking of amyloid precursor protein. J Neurosci 33, 64-71, https://doi.org/10.1523/JNEUROSCI.2371-12.2013 (2013).

24. Mazella, J. et al. The 100-kDa neurotensin receptor is gp95/sortilin, a non-G-protein-coupled receptor. J Biol Chem 273, 26273-26276, https://doi.org/10.1074/jbc.273.41.26273 (1998).

25. Gustafsen, C. et al. The hypercholesterolemia-risk gene SORT1 facilitates PCSK9 secretion. Cell Metab 19, 310-318, https://doi. org/10.1016/j.cmet.2013.12.006 (2014).

26. Pan, X., Zaarur, N., Singh, M., Morin, P. \& Kandror, K. V. Sortilin and retromer mediate retrograde transport of Glut4 in 3T3-L1 adipocytes. Molecular biology of the cell 28, 1667-1675, https://doi.org/10.1091/mbc.E16-11-0777 (2017).

27. Xu, W. et al. The genetic variation of SORCS1 is associated with late-onset Alzheimer's disease in Chinese Han population. PLoS One 8, e63621, https://doi.org/10.1371/journal.pone.0063621 (2013).

28. Suh, J. et al. ADAM10 missense mutations potentiate beta-amyloid accumulation by impairing prodomain chaperone function. Neuron 80, 385-401, https://doi.org/10.1016/j.neuron.2013.08.035 (2013).

29. Cariou, B. et al. PCSK9 dominant negative mutant results in increased LDL catabolic rate and familial hypobetalipoproteinemia. Arterioscler Thromb Vasc Biol 29, 2191-2197, https://doi.org/10.1161/ATVBAHA.109.194191 (2009).

\section{Acknowledgements}

The authors acknowledge the facilities, and the scientific and technical assistance of Sydney Microscopy and Microanalysis, University of Sydney and Sydney Mass Spectrometry, Charles Perkins Centre. The work was supported by Diabetes Australia Research Program (Y16G-KEBM). MAK is supported by a Jennie Mackenzie Philanthropic Fellowship, University of Sydney. Y.A. is recipient of the Australian Postgraduate Scholarship. ZB was a recipient of Charles Perkins Centre Summer Scholarship.

\section{Author contributions}

M.A.K. conceived and designed the study. B.Y. and Z.B. performed the majority of the experiments. Y.A. performed deglycosylation experiments. Z.S. performed two of the LC-MS/MS experiments. All authors performed data analysis and statistical analysis for the respective experiments they performed. B.Y. and M.A.K. wrote the paper. All authors reviewed and edited the manuscript and approved the final version of the manuscript.

\section{Competing interests}

The authors declare no competing interests.

\section{Additional information}

Supplementary information is available for this paper at https://doi.org/10.1038/s41598-019-55873-6.

Correspondence and requests for materials should be addressed to M.A.K.

Reprints and permissions information is available at www.nature.com/reprints.

Publisher's note Springer Nature remains neutral with regard to jurisdictional claims in published maps and institutional affiliations.

Open Access This article is licensed under a Creative Commons Attribution 4.0 International License, which permits use, sharing, adaptation, distribution and reproduction in any medium or format, as long as you give appropriate credit to the original author(s) and the source, provide a link to the Creative Commons license, and indicate if changes were made. The images or other third party material in this article are included in the article's Creative Commons license, unless indicated otherwise in a credit line to the material. If material is not included in the article's Creative Commons license and your intended use is not permitted by statutory regulation or exceeds the permitted use, you will need to obtain permission directly from the copyright holder. To view a copy of this license, visit http://creativecommons.org/licenses/by/4.0/.

(c) The Author(s) 2019 